Supporting Information (SI)

\title{
Polarization of Stem Cells Directed by Magnetic- Field-Manipulated Supramolecular Polymeric Nanofibers
}

Bing Zhang, Qilin Yu, and Yu Liu*

B. Zhang, Y. Liu

College of Chemistry, State Key Laboratory of Elemento-Organic Chemistry, Nankai University, Tianjin 300071, China; Collaborative Innovation Center of Chemical Science and Engineering (Tianjin), Tianjin 300072, China.

E-mail: yuliu@nankai.edu.cn

Q. Yu

Key Laboratory of Molecular Microbiology and Technology, Ministry of Education, College of Life Sciences, Nankai University, Tianjin 300071, China. 


\section{Figures}

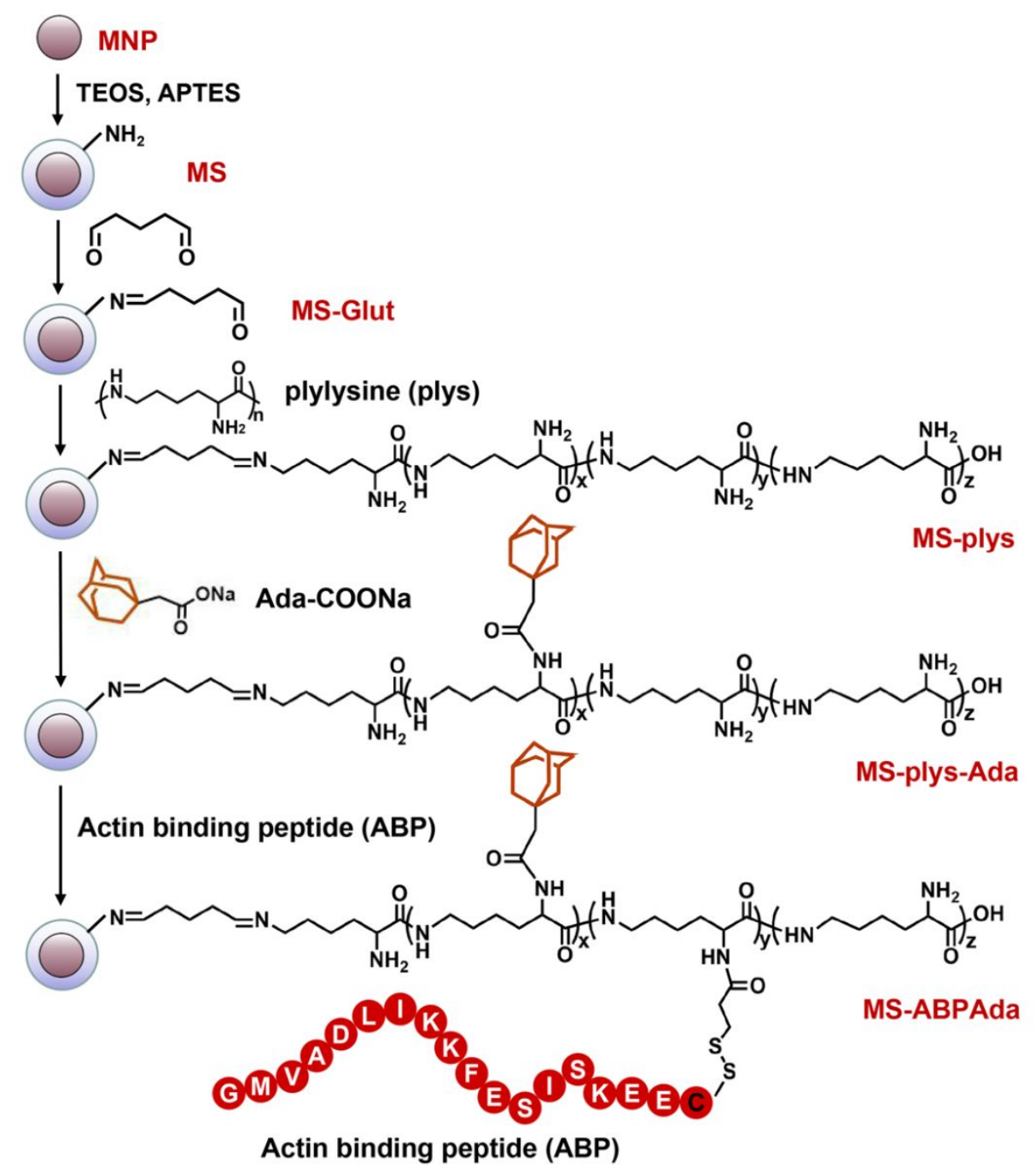

Figure S1. Synthetic route of MS-ABPAda used in this study.

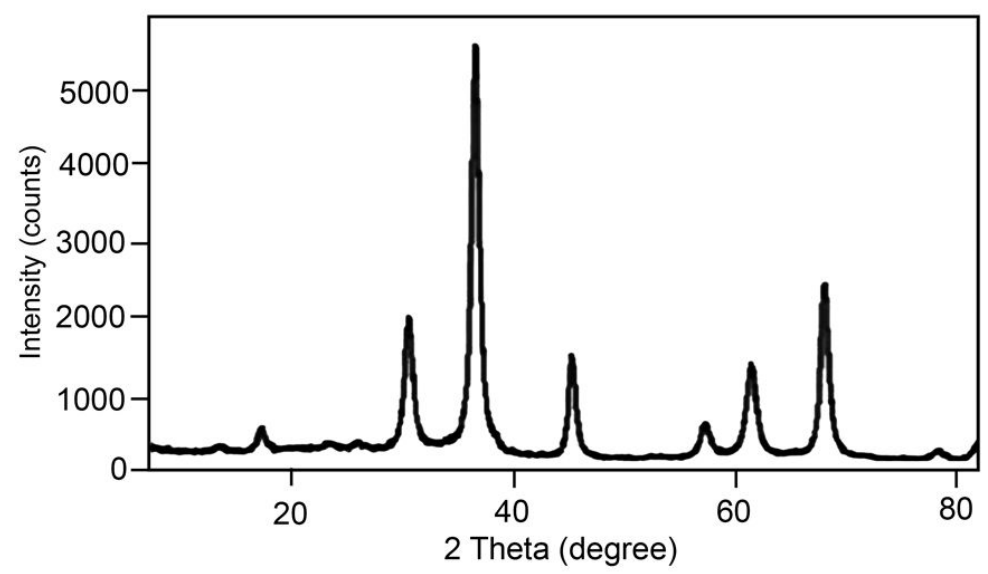

Figure S2. XRD spectrum of the synthesized $\mathrm{Fe}_{3} \mathrm{O}_{4}$ nanoparticles. 

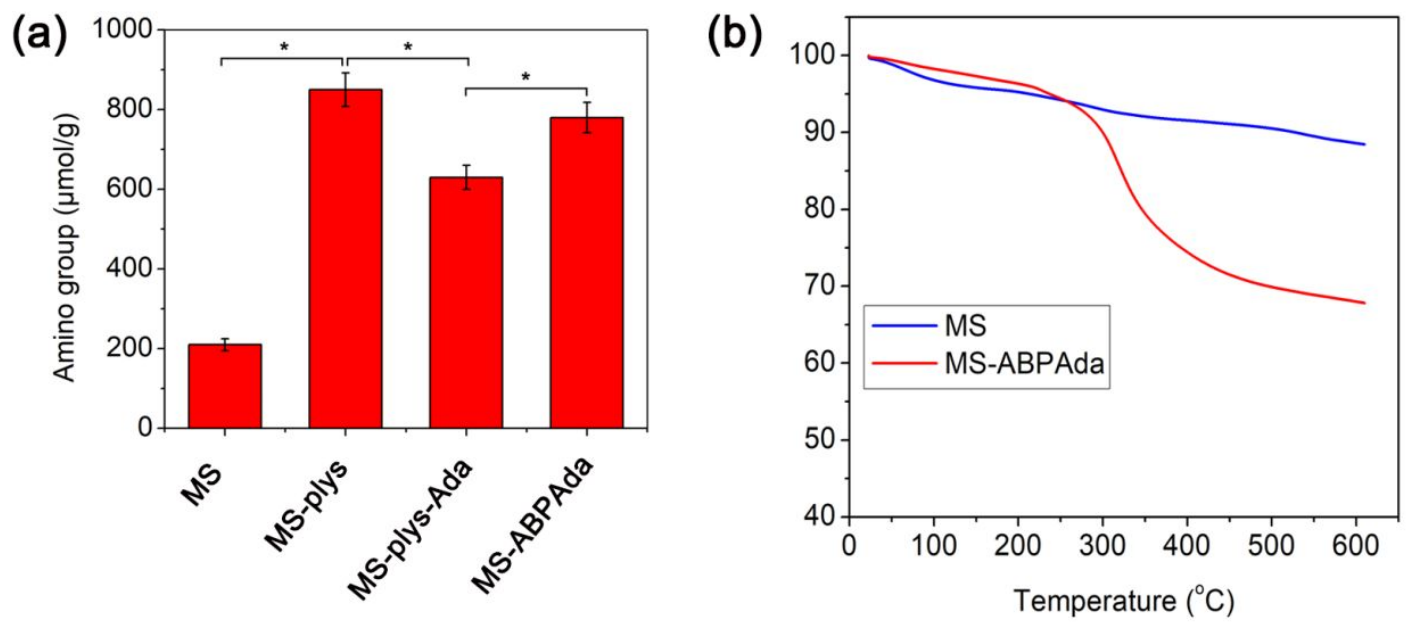

Figure S3. Contents of amino group in the synthesized nanoparticles revealed by the SPDP method (a) and thermogravimetric curves of the nanoparticles (b). * indicates significant difference between the groups $(P<0.05)$.

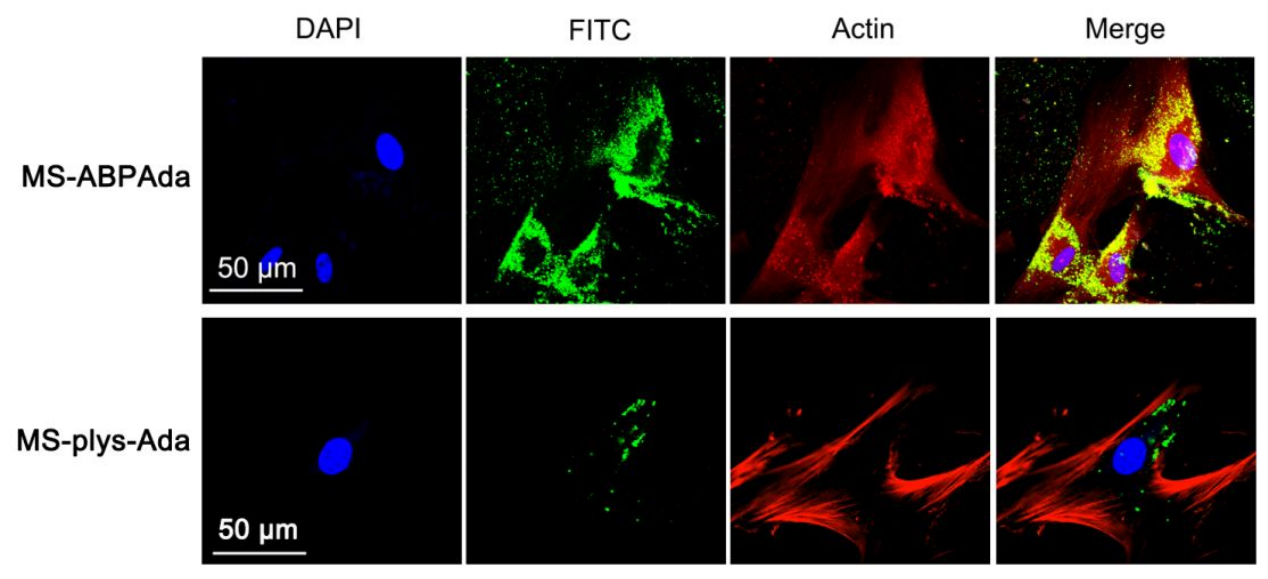

Figure S4. Localization of MS-ABPAda (up) rather than MS (down) at the actin cytoskeleton. The NPs (100 mg/L) were added into the culture dishes of DPSCs. The cells were cultured for $24 \mathrm{~h}$, fixed by formaldehyde, and stained by rhodamine B-phalloidin ( 5 $\mathrm{mg} / \mathrm{L}$ ) and DAPI ( $5 \mathrm{mg} / \mathrm{L})$. The stained cells were then observed by confocal microscopy. Noting that MS-ABPAda could be co-localized with actin cytoskeleton, while FITC-tagged MS could not. 

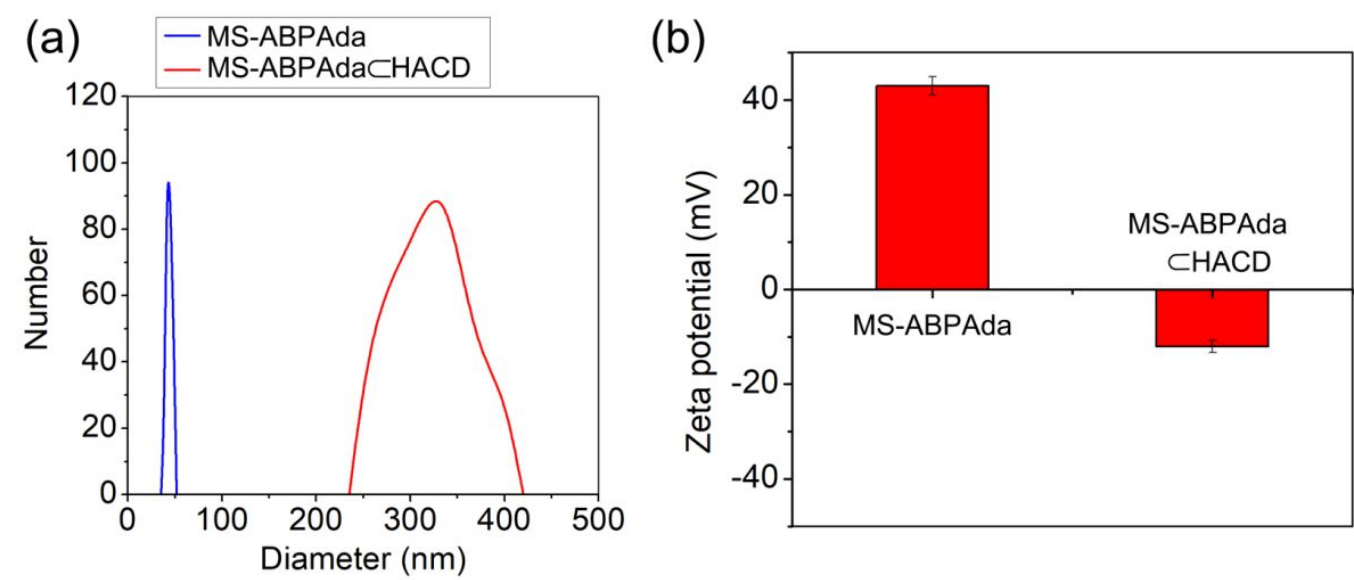

Figure S5. Size distribution (a) and Zeta potential (b) of MS-ABPAda and the MSABPAdacHACD assemblies.

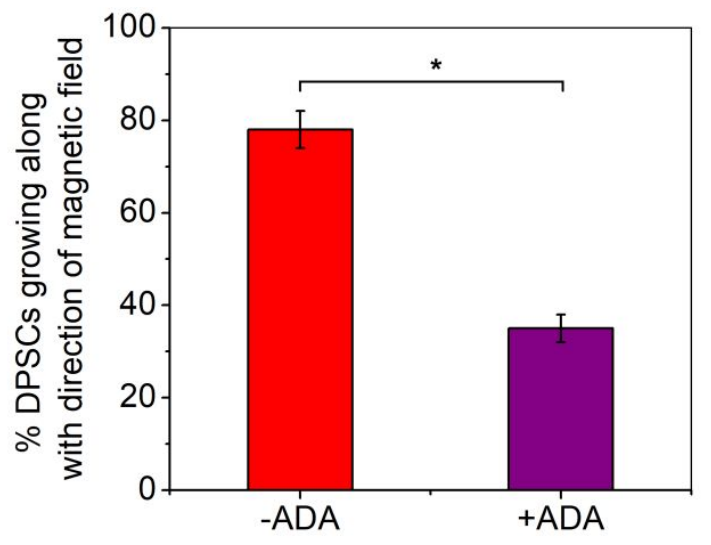

Figure S6. Attenuation of directed growth of DPSCs by adamantane-1-acetic acid, sodium salt (1 mM) (ADA, $1 \mathrm{mM})$.

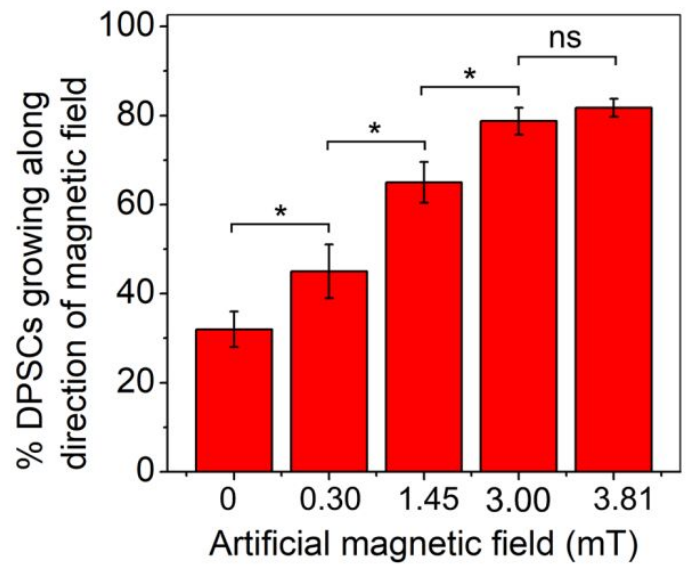

Figure S7. Percent of the nanofiber-treated DPSCs growing with the direction of magnetic field at different strengths. * indicates significant difference between the groups $(P<0.05)$. 


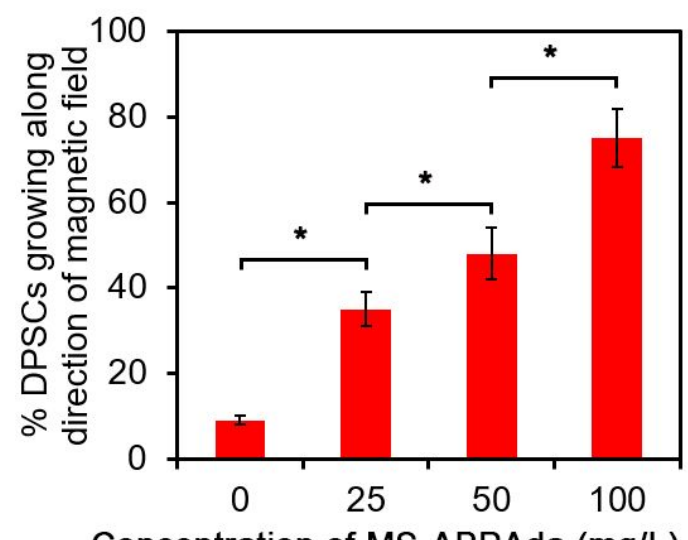

Concentration of MS-ABPAda (mg/L)

Figure S8. Percent of the nanofiber-treated DPSCs growing with direction of magnetic field at different concentrations of MS-ABPAdaCHACD assemblies (MS-ABPAda at indicated concentrations and HACD with the corresponding equal concentrations). * indicates significant difference between the groups $(P<0.05)$.

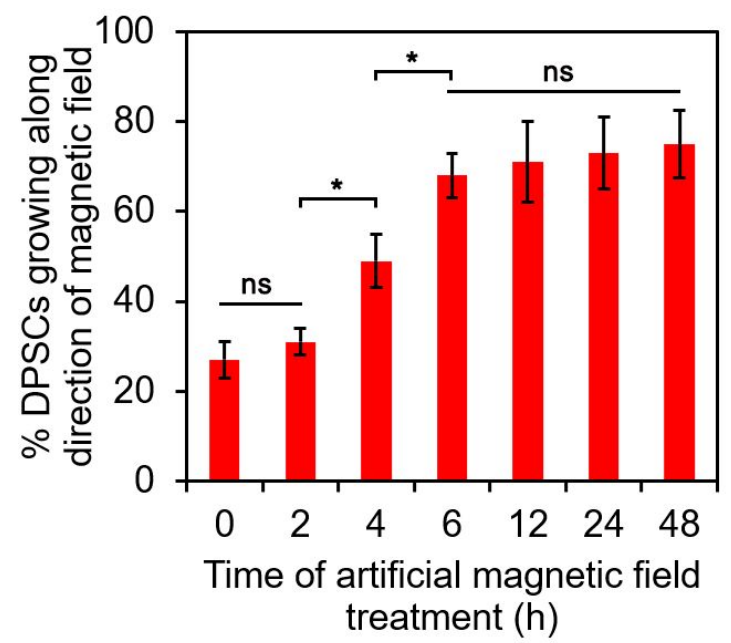

Figure S9. Percent of the nanofiber-treated DPSCs growing with direction of magnetic field with different time of artificial magnetic field treatment $(3 \mathrm{mT}) .{ }^{*}$ indicates significant difference between the groups $(P<0.05)$. 


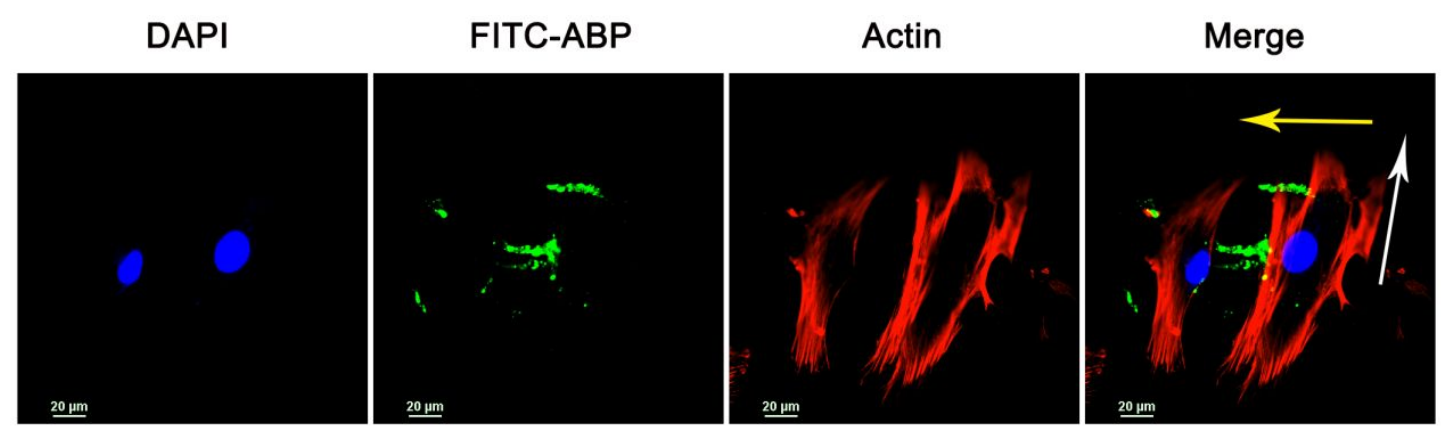

Figure S10. Resistance of pre-formed cellular polarization to change of magnetic field direction. The DPSCs containing the MS-ABPAdacHACD assemblies were pre-treated magnetic field with the direction indicated by the white arrows for $24 \mathrm{~h}$, followed by treatment of magnetic field with the direction indicated by the yellow arrows for another 24 h. The cells were then fixed and stained for confocal microscopy. Noting that the preformed cellular polarization was not changed by alteration of the second magnetic field.

(a)

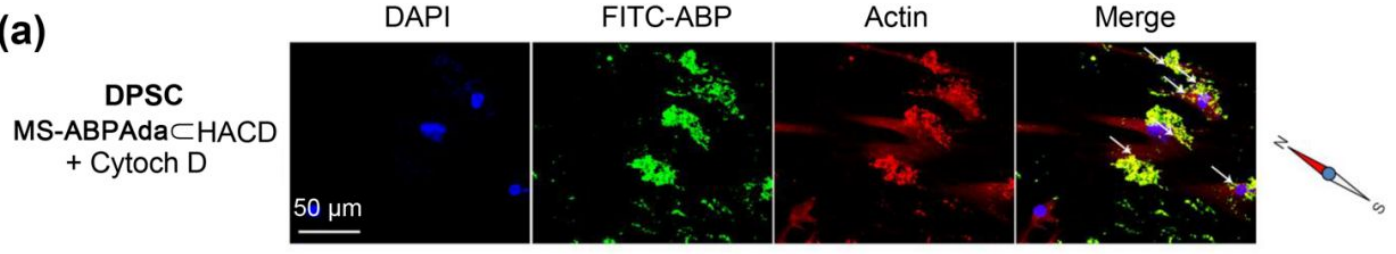

(b)

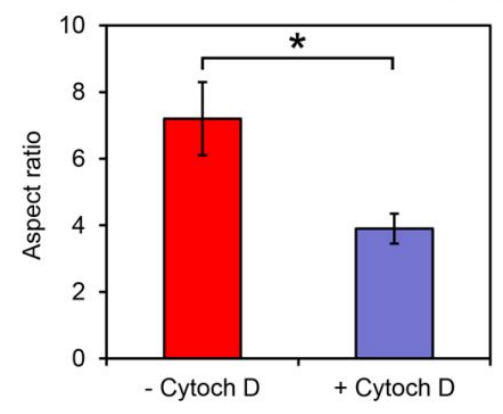

(c)

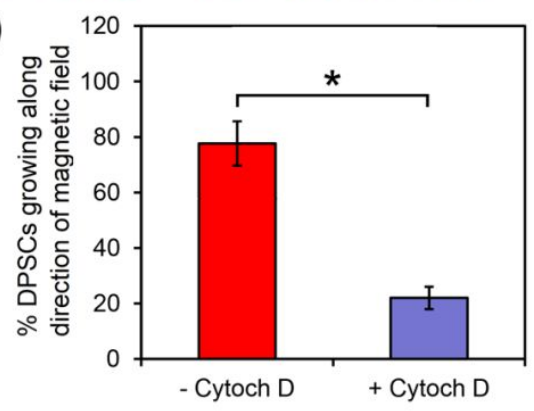

Figure S11. Attenuation of morphology and directed growth of the assembly-treated DPSCs by the actin cytoskeleton disruptor cytochalasin D (Cytoch D). (a) Confocal images of the DPSCs treated by MS-ABPAdaCHACD and Cytoch D (MS-ABPAda $100 \mathrm{mg} / \mathrm{L}$, HACD $100 \mathrm{mg} / \mathrm{L}$, Cytoch D $2 \mu \mathrm{M})$ under artificial magnetic field $(3 \mathrm{mT})$ for $48 \mathrm{~h}$. (b) Aspect ratio of the treated cells. ${ }^{*}$ indicates significant difference between the groups $(P<0.05)$. (c) Percent of DPSCs growing treated by MS-ABPAdacHACD and Cytoch D along with the direction of the artificial magnetic field at $3 \mathrm{mT} .{ }^{*}$ indicates significant difference between the groups $(P<0.05)$. 

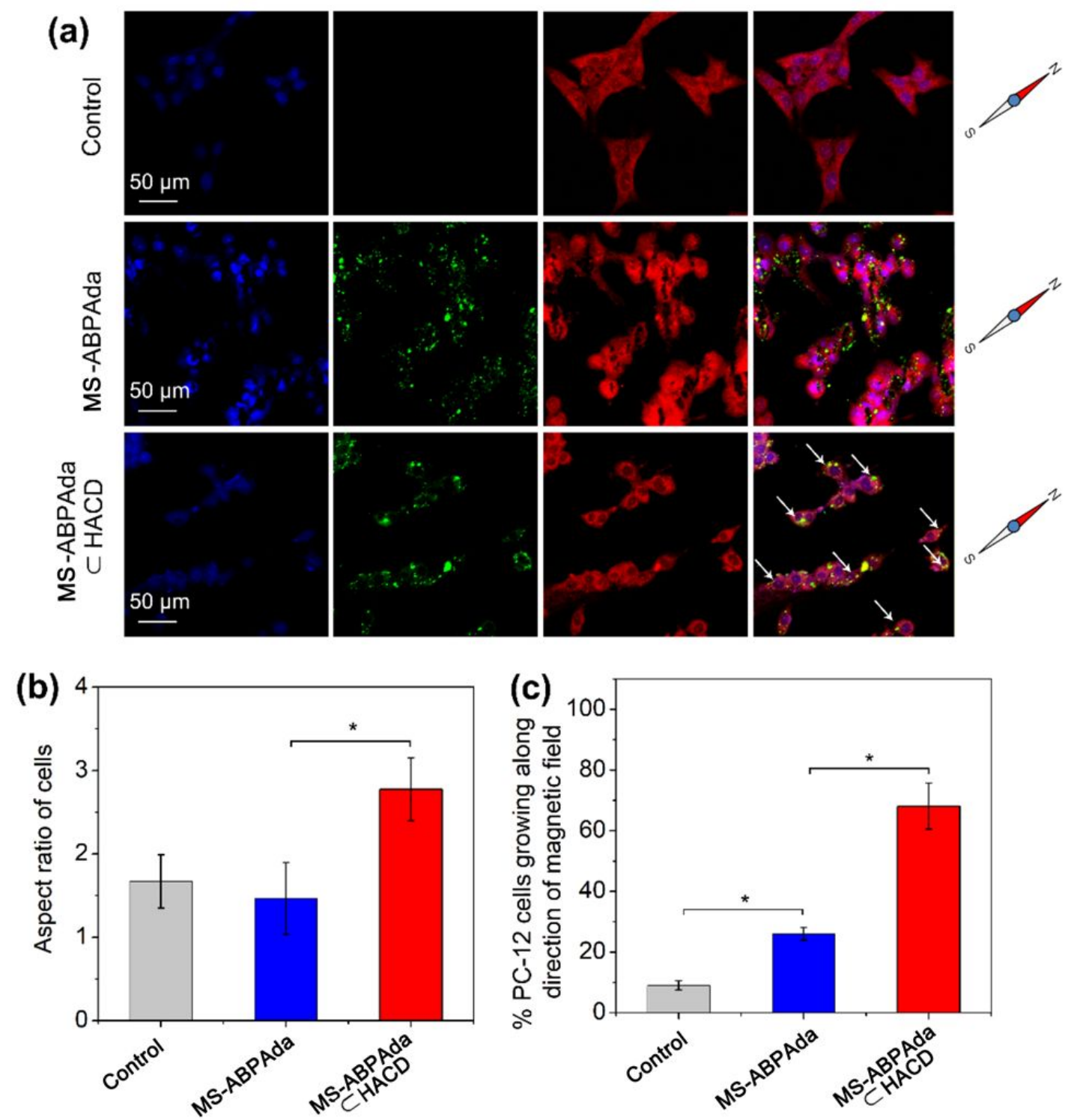

Figure S12. Magnetic field-directed polarization of PC-12 cells by the MS-ABPAdacHACD nanofibers after $48 \mathrm{~h}$ incubation. (a) Confocal images of PC-12 cells treated by the nanocomposites under the artificial magnetic field $(3 \mathrm{mT})$. The white arrows indicated the polarized cells. (b) Aspect ratio of PC-12 cells. (c) Percent of PC-12 growing along with the direction of the artificial magnetic field at $3 \mathrm{mT}$. * indicates significant difference between the groups $(P<0.05)$. 


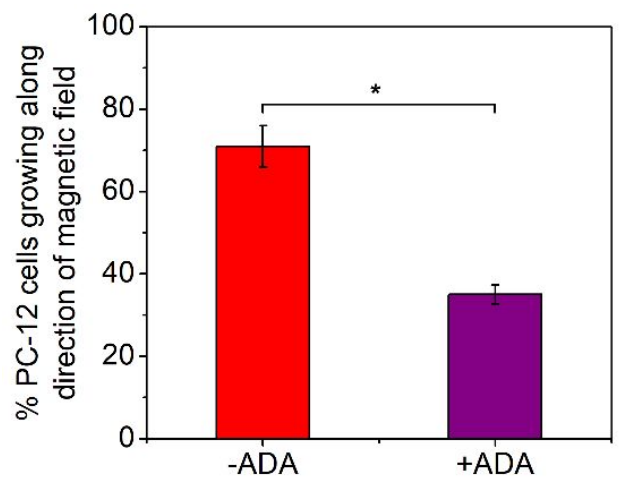

Figure S13. Attenuation of directed growth of PC-12 cells by adamantane-1-acetic acid, sodium salt (ADA, $1 \mathrm{mM})$.

(a)

$$
\begin{gathered}
\text { PC-12 } \\
\text { MS-ABPAda } \subset \text { HACD } \\
+ \text { Cytoch D }
\end{gathered}
$$
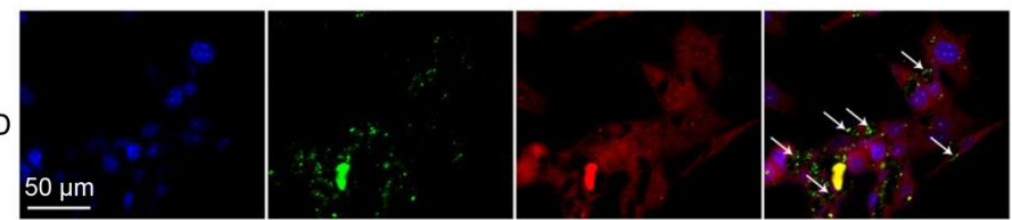

(b)
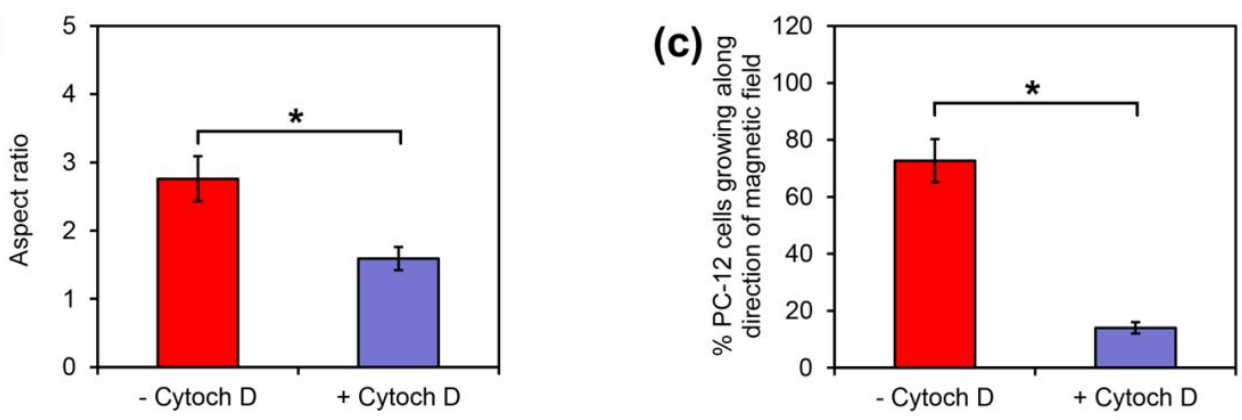

Figure S14. Attenuation of morphology and directed growth of the assembly-treated PC12 cells by cytochalasin $\mathrm{D}$ (Cytoch $\mathrm{D}$ ). (a) Confocal images of the PC-12 treated by MSABPAdacHACD and Cytoch D under artificial magnetic field (3 mT) for $48 \mathrm{~h}$. (b) Aspect ratio of the treated cells. * indicates significant difference between the groups $(P<0.05)$. (c) Percent of PC-12 growing treated by MS-ABPAdacHACD and Cytoch $D$ along with the direction of the artificial magnetic field at $3 \mathrm{mT}$. * indicates significant difference between the groups $(P<0.05)$. 
(a)

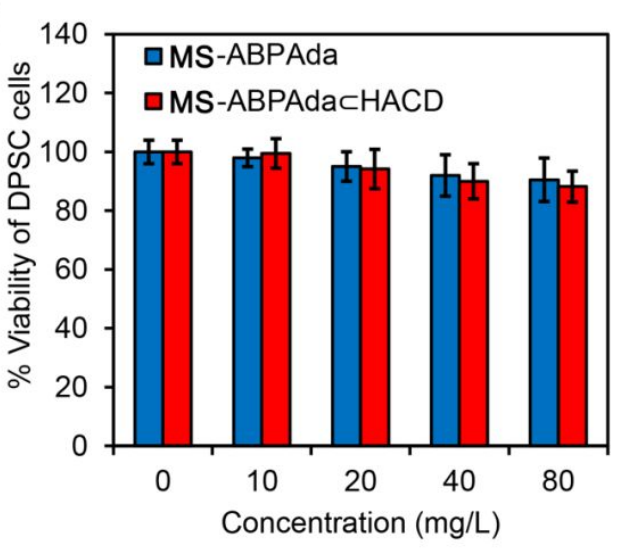

(b)

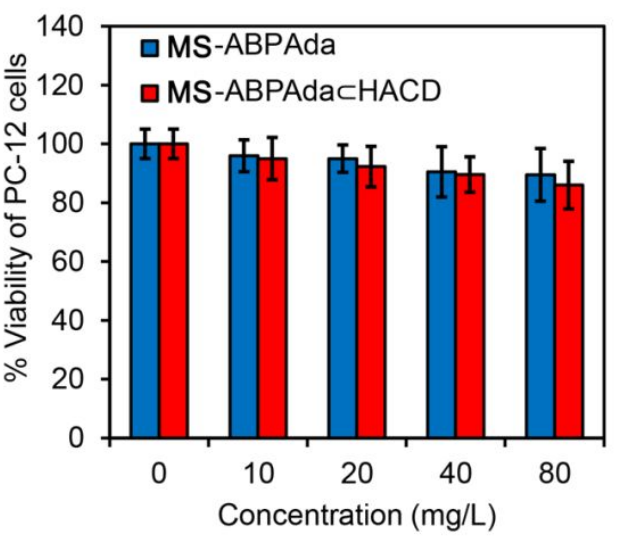

Figure S15. Viability of DPSCs (a) and PC-12 cells (b) under treatment of MS-ABPAda or MS-ABPAdaCHACD in the presence of artificial magnetic field $(3 \mathrm{mT})$. 